\title{
THE STUDY OF COMPLICATIONS OF ND: YAG LASER CAPSULOTOMY
}

\section{GORE V.S.}

MGM Medical College, Navi Mumbai, MS, India

${ }^{*}$ Corresponding Author: Email- drvarshavgore@gmail.com

\begin{abstract}
-
Objective- To study the complications of ND:YAG Laser capsulotomy.

Participants- Patients between age group of 08 to 80 who is having significant posterior capsular opacification leading to decreased visual acuity from early post operative days.

Material and Method- 200 patients with PCO were studied for post ND:YAG laser complications. The patient who is not having any organic cause of decreased vision and who have completed at least three months after cataract surgery were selected. ND:YAG laser procedure was performed and their visual acuity improvement along with complications associated with this procedure were studied.

Results- The procedure is absolutely safe if strict selection (inclusion/exclusion) criteria is followed. Most of complications are transient which can be managed by proper medication. Complications like retinal detachment or cystoid macular edema are multifactoral and can not always be only due to laser capsulotomy alone. Endophthalmitis is rare complication but highly unpredictable which can not be ruled out but be well treated with newer generation intravitreal drugs or vitrectomy surgery. Most of the patients $86 \%$ were significantly benifited by improved visual acuity.
\end{abstract}

Key words- ND: YAG Laser Capsulotomy, cystoid macular edema, Endophthalmitis, Optic atrophy, Amblyopia, Corneal dystrophydegeneration, iriti's.

Abbreviations- PCO ( Posterior capsular opacification),IOL (Intraocular lens)

Citation: Gore V.S. (2012) The Study of Complications of ND: YAG Laser Capsulotomy. International Journal of Bioinformatics Research, ISSN: 0975-3087 \& E-ISSN: 0975-9115, Volume 4, Issue 2, pp.-265-268.

Copyright: Copyright@2012 Gore V.S.. This is an open-access article distributed under the terms of the Creative Commons Attribution License, which permits unrestricted use, distribution, and reproduction in any medium, provided the original author and source are credited.

\section{Introduction}

Posterior capsular opacification (PCO) also called as after cataract is a nagging post surgical complication following phacoemulsification or non phacoemulsification cataract surgery with posterior chamber intraocular lens implantation.

The term posterior capsular opacification is actually a misnomer.It is not the capsule which opacifies,rather an opaque membrane develops as retained cells proliferate and migrate on the posterior capsular surface.

Though there are many factors suggested to reduce posterior capsular opacification. The incidence of posterior capsular opacifi- cation still exists considerably. And till today ND:YAG laser capsulotomy has been the ultimate choice to get rid of it.

The nd:yag lasing medium is a man made crystal of Neodymium doped yttrium-Aluminium-Garnet is a photodisrupter not a photocoagulator like argon or krypton laser[1].Laser has range of applications in ophthalmology in anterior \& posterior segment on various parts[2].In laser machines, emitted photons are trapped in highly polished mirrors forcing them to travel back \& forth in the cavity. When a photon passes close to an excited particle, the particle will be stimulated to emit a photon that is identical in wavelength, phase and spatial coherence to the first. This amplifi- 
cation continues, increasing the number of active photons[3,4]. The laser power setting is between 1 to $2.5 \mathrm{mj} /$ pulse or if mode is locked then between 3 to $5 \mathrm{mj} / \mathrm{pulse}$. The capsulotomy is performed by series of punctures in cruciate pattern with first puncture aimed at visual axis. An opening about $3 \mathrm{~mm}$ is usually adequate but larger might be needed for peripheral retinal visualization and its treatment [5].

\section{Aims and Objective}

1. To study the intra \& postoperative complications of ND: YAG laser posterior capsulotomy.

2. To study incidence of complications.

3. To study the visual improvement (outcome).

\author{
Materials and Method \\ Inclusion Criteria \\ 1. Significant PCO. \\ 2. Good immediate post (cataract) operative visual acuity. \\ 3. No corneal /retinal (organic) pathology.
}

\section{Exclusion Criteria}

1. less than 3 months post cataract surgery.

2. Amblyopia.

3. Optic atrophy.

In this study 200 patients with significant posterior capsular opacification were treated with ND:YAG laser and followed up from period of June 2010 to December 2011.

The criteria for selection of patient was significant posterior capsular opacification causing decrease in visual acuity. No attempt has been made to limit the study to specific age group or gender.

In all patients a complete ophthalmic history including history of timing of cataract surgery, history of any preexisting glaucoma, retinal pathology, amblyopia, opticatrophy, cornealopacity, Corneal dystrophy-degeneration or any other ocular condition was recorded. History of any topical or systemic medication and other significant systemic illness was also recorded.

Visual acuity for distant and near was recorded before and after the procedure. Anterior segment examination and intraocular pressure measuring on applanation tonometry was done with slit lamp. Direct and indirect ophthalmoscopy was performed to rule out any pre existing macular or retinal pathology. After recording all findings laser capsulotomy was performed in all suitable patients.

Following procedure was performed for performing YAG laser capsulotomy in the present study.

The purpose, procedure and possible complications were explained to every patient and their relatives in best possible language. Written informed consent was taken in each case.

Routinely dilatation before laser application was not required but it was preferred in cases of dense PCO and in uncoopertive cases. If the pupil was to be dilated then the landmarks of the pupillary zone of capsule was sketched to avoid eccentric opening. The middle of the pupillary axis was marked by a single marker shot. Patients head position was adjusted in such a way that chin and forehead would rest well at its respective position. A drop of topical anaesthetic proparacaine hydrochloride ophthalmic solutionUSP0.5\%w/v was instilled before the procedure. A contact lens called modified Abraham lens was used whenever necessary.
Capsule was examined for wrinkles and tension lines, because if shots were fired across tension lines it might lead to larger and uncontrolled opening per pulse.

For accurate focusing, the intersection of the He-Ne beam where the two red spots becomes one that spot was used. It locates the focal point of laser energy. For energy settings we began with less energy $1 \mathrm{mj}$ at monoburst and increased in steps as required until tissue breakdown was achieved. Successive shots were fired to make opening ' $X$ ' or ' $A$ 'shaped. If the membrane did not open focal point was shifted slightly forward.

Usually $3 \mathrm{~mm}$ opening was made which was adequate for good visual acuity. Following the procedure all patients were routinely given topical antibiotic-steroid combination drops and topical antiglaucoma drops. Patients were followed up on day one, day seven, day fourteen, one month then after six months interval. On each follow up patients were examined for visual acuity for distant and near. Intraocular pressure measured, slit lamp examination and fundoscopy was done. Rise of intra ocular pressure was noted and labeled as immediate rise, if the rise of IOP (intra ocular pressure) was for hours and returned to normal at end of seven days and Persistent rise if sustained high IOP was measured on subsequent follow up.

\section{Results}

Out of 200 patients number of male patients were 123 meaning $61.5 \%$ and female 77 meaning $38.5 \% .13$ patients had to undergo repeated YAG due to inadequate opening. 10 out of which were below 20 years of age because of thicker PCO in younger age group is very common.

\section{Age Distribution}

Table 1-

\begin{tabular}{|ll|}
\hline Age Group & Total Cases \\
$08-30$ & 07 \\
$31-40$ & 13 \\
$41-50$ & 89 \\
$51-60$ & 73 \\
$61-70$ & 15 \\
$71-80$ & 03 \\
\hline
\end{tabular}

\section{Sex Distribution}

\begin{tabular}{|lll|}
\hline Total Cases & Male & Female \\
\hline 200 & 123 & 77 \\
100 & $61.5 \%$ & $38.5 \%$ \\
\hline
\end{tabular}

\section{Pre YAG Vision}

Table 3-

\begin{tabular}{|lll|}
\hline Pre YAG Vision & No. of Cases & Percentage \\
\hline $6 / 12$ & 56 & $28 \%$ \\
$6 / 18$ & 67 & $33.5 \%$ \\
$6 / 24$ & 49 & $24.5 \%$ \\
$6 / 36$ & 17 & $8.5 \%$ \\
$6 / 60$ & 9 & $4.5 \%$ \\
Finger Counting & 2 & $1 \%$ \\
\hline
\end{tabular}

For all patients initially energy was kept at $1 \mathrm{mj}$ and gradually increased in step till desired result was obtained. Burst was kept monoburst. Majority of patients had adequate opening with shots between 6 to 12.Veryfew patients required more than 12 shots. 


\section{Post YAG Vision}

Table 4-

\begin{tabular}{|lll|}
\hline Vision & No. of Cases & Percentage \\
\hline $6 / 18-6 / 6$ & 183 & $91.5 \%$ \\
6.24 OR LESS & 17 & $8.5 \%$ \\
\hline
\end{tabular}

\section{Complications}

Table 5-

\begin{tabular}{|llll|}
\hline Sr. No. & Complications & No. of Cases & Percentage \\
\hline 1. & CORNEAL INJURY & 2 & $1 \%$ \\
2. & IRITIS & 67 & $33.5 \%$ \\
3. & HYPHEMA & 3 & $1.5 \%$ \\
4. & IOL RELATED & & \\
& a)pitting & 9 & $4.5 \%$ \\
& b)cracks & 3 & $1.5 \%$ \\
& c)decentration & 3 & $1.5 \%$ \\
5. & IOP RISE & & \\
& a)Immediate & 79 & $39.5 \%$ \\
6. & b)persistent & 7 & $3.5 \%$ \\
7. & Cystoid macular edema & 8 & $4 \%$ \\
8. & Retinal damage & 1 & $0.5 \%$ \\
\hline
\end{tabular}

\section{Discussion}

Since the advent of YAG in 1981 its application ranged remarkably. Its acceptance in the management in PCO was well appreciated in surgeons as well as patients due to the non surgical and non invasive approach as against needling which was mainstay in PCO management prior to YAG [6].

Complications seen during procedure are discussed below

In complications related to IOL (Intraocular lens) i.e. pitting, cracks or decentration none of the IOL'S were glass or silicon. All were made up of polymethylmethacrylate (PMMA). More pitting was seen in cases of dense PCO with more number of shots in uncooperative patients. Such was more observed in pediatric age group or with intraocular lenses having deposits or scratches due to surgery.

There was no decrease or disturbance in visual acuity with only pitting of IOL but $1.5 \%$ who had cracks in IOL failed to improve more than 6/18.It was observed that using monoburst and avoiding multiple burst modes and by using minimum energy we could reduce IOL damage. Use of contact lens stabilizes the eye, increases convergence and divergence angle which lowers the retinal side effects and improves laser beam effect. Optics acts as a heat sink and facilitates accurate focusing and magnification [7]. Pitting of IOL can also be reduced by deep focusing technique where optical break down occurs in the anterior vitreous and the shock wave radiate forward and ruptures the capsule.

Postcapsulotomy intraocular pressure elevation is a common but transient complication following this procedure. In most cases IOP returned to pretreatment level within 10days. $3.5 \%$ had persistent rise of IOP on subsequent follow up, so topical pressure reducing agent were continued for longer time. Antiglaucoma medication was reduced gradually and finally omitted. One patient with preexisting glaucoma had exacerbation, mean IOP before capsulotomy was $23 \mathrm{~mm}$ hg and mean IOP during first week of post laser was $30 \mathrm{~mm}$ hg. After 6 months the mean IOP was 28. Patient whose IOP was uncontrolled had to undergo trabeculectomy surgery. It was observed that having rise in IOP in absence of PCIOL and use of higher total energy is related to posterior trapping of capsular debries [8].The acute pressure rise is caused by impaired aqueous outflow and rapid onset suggests that the reduced outflow mostly due to capsular debris, acute inflammatory cells, heavy molecular weight protein or a combination of these mechanisms $[9,10]$. The IOP rise was correlated with previously high IOP. Higher the pretreatment IOP leads to greater the chance of high pressure rise. Therefore glaucoma patients were at higher risk of developing pressure elevation. Also the presence of PCIOL may result in less incidence of transient IOP rise but it does not necessarily protect against the onset of persistent IOP elevation [11].

Four percent $(4 \%)$ of patients had cystoids macular edema and were treated with topical anti-inflammatory drugs for 21 days. Visual acuity improved slightly but failed to improve more than $6 / 18$. This is more seen in patients who had greater inflammatory reaction in their cataract operation. One of this patient had preexisting diabetic retinopathy. The development of retinal complication remains more in a patient who had grater inflammatory reaction to surgery [12].

In this study none of the patients had retinal complications like tear, hole or detachment. Thirty three point five percent (33.5\%) of patient had iritis after YAG capsulotomy manifested as cells and flare in the anterior chamber on slit lamp examination. They were given topical steroid and reaction had subsided leaving no delayed complication. Corneal injury was seen in one percent $1 \%$ of patient and hyphema seen in $1.5 \%$ cases. The damage to cornea or iris can occur due to misfiring of shots. In cornea it causes stromal haze but clears within 3 days. Hyphema also gets resolved within 3 to 7 days depending on size without any treatment and complications.

\section{Conclusion}

There are many methods tried for prevention of PCO but YAG laser capsulotomy remains the most common and safe procedure. When correctly done with proper patient selection and thorough fundus examination and at least after three months after cataract surgery. It is very economical, convenient, fast and non invasive OPD procedure with immediate results which is easily mastered with some practice. Although non invasive and generally considered safer it carries a low but finite risk of complications. These complications are rare and rarely sight threatening.

Therefore we should be alert of development of cystoids macular edema, retinal detachment. Persistent rise in IOP during follow up of patients with ND: YAG laser posterior capsulotomy.

\section{References}

[1] Francis L' Esperence (1983) Neodynium-YAG laser, Ophthalmic lasers. Mosby, 2nd edition, 79-83.

[2] Spansrov A.F. (1981) ND:YAG Laser in ophthalmology, Laser and their applications, 129-132.

[3] Thomos D. Duane. An introduction to basic laser physics. Clinical ophthalmology. Harper and raw publisher, clinical volume -1 chapter-69, 390-98.

[4] Marshall J. (1982) Properties of laser light, Laser in ophthalmology Basic principal, 20-26

[5] Jack J. Kanski (2003) Posterior capsular opacification. Clinical ophthalmology. Butterworth- Einemann, fifth international edi- 
tion, 180-181.

[6] Peyman G.A., Sanders D.R., Goldberg M.F. (1987) Opacification of the posterior capsule, Principles and practice of ophthalmology Jaypee., 1 st edition, 2, 617-618.

[7] Albert D.M., Jackobiec F.A. (1994) Use of contact lens for laser delivery, Principle and practice of Ophthalmology, 3, 1600.

[8] Channel M.M., Beckman H. (1984) Archives of ophthalmology 102 (4), 1024-1026.

[9] Richter C.U., Arzeno G., Pappas R. (1985) Archives of ophthalmology, 103(7), 912-915.

[10]Gimbel H.V., Van Western Brugge, Sanders J.A. (1990) Archives of ophthalmology, 108(8), 1126-1129.

[11]Fourman S., Apison J. (1991) Archieves of ophthalmology, 109(4), 511-513.

[12]Steinert R.F., Puliatio C.A., Kumar S.R., Dvdek S.D. (1991) American journal of ophthalmology, 112(4), 373-80. 